\title{
An inhalation anesthetic device for stereotaxic operation on mouse pups
}

Sachine Yoshida, Yuya Morimoto, Taishi Tonooka, Shoji Takeuchi

Institute of Industrial Science, The University of Tokyo, 4-6-1 Komaba, Meguro-ku, Tokyo 153-8505, JAPAN

\section{Keywords}

3D printer, CAD data, PDMS, tracer injection

\begin{abstract}
Abbreviations
BDA, biotynilated dextran amine; BLP, basolateral amygdaloid nucleus posterior part; CAD, computer aided design; LEnt, lateral entorhinal cortex; PDMS, polydimethylsiloxane; PMCo, posteromedial cortical amygdaloid nucleus; PND, postnatal day; Py, pyramidal tract
\end{abstract}

\begin{abstract}
Background: Mouse pups are invaluable model animals for understanding the molecular and neural basis underlying behavioral development. Stereotaxic operations with anesthetic control are useful tools in systems neuroscience. However, there are no commercially available anesthetic or stereotaxic devices for mouse pups. Current devices have several problems such as invasive approach for stabilization, poor sanitary control, and less flexibility to combine other surgical apparatuses.

New Method: Here, we developed an inhalation anesthetic device equipped with stereotaxic function for mouse pups, by using polydimethylsiloxane (PDMS). PDMS is tolerant to heat and water exposure, and soft enough to cut or make a hole. The anesthetic and the stereotaxic parts were fabricated from the three-dimensional computer-aided design (3D CAD) data obtained from the head of a real mouse pup.
\end{abstract}


Results: To confirm its utility, a tracer was injected into the brain. We were able to anesthetize and stabilize pups at once in a non-invasive manner using the PDMS device. The histological staining revealed that tracer injection was successful. Our device was compatible with various types of commercial stereotaxic and anesthetic apparatuses via trimming and tube insertion, respectively.

Comparison with Existing Method(s): To our knowledge, this is the first report of a device that can stabilize the mouse pup's head with the non-invasive manner and functions as an inhalation anesthetic device that can be sterilized.

Conclusions: The present fabrication method will provide a handy and functional instrument for stereotaxic operations in animal models at various developmental stages.

\section{Introduction}

Mice are invaluable experimental animals for understanding the behavioral regulation at the molecular level. In the experimental systems neuroscience, stereotaxic surgical operations in adult mice are highly effective and have various applications such as site-specific brain lesions, targeted delivery of chemicals and vectors, and implantation of electrodes (Tsuneoka et al., 2013; Cetin et al., 2007). To perform these operations, adult mice are often anesthetized using barbiturates, and stabilized using commercially available stereotaxic apparatus. However, the stereotaxic operations in mouse pups that provide valuable information in the neural basis of behavioral development have technical difficulties in stabilizing the head and in controlling the dosage of the barbiturates. In pups, it is impossible to stabilize the head by using ear bars, since the external auditory meatus that accepts the ear bars in the adult are not fully opened yet. The skull is too fragile to stabilize tightly. In addition, the dosage control of barbiturate anesthesia is hard, unlike adult mice, because immature animals have lower levels of serum albumin and body fat, which contribute to the diminished efficacy of the barbiturates (Flecknell, 1987). Consequently, there are no commercial inhalation anesthetic devices or stereotaxic instruments specialized for mouse pups that can stabilize the head in a non-invasive manner.

In this paper, we develop an inhalation anesthetic device equipped with stereotaxic function. To stabilize the pup's head in a non-invasive manner, we employ three-dimensional (3D) computer-aided design (CAD) data of an actual pup head and develop the anesthetic and stereotaxic parts. The present device consists of polydimethylsiloxane (PDMS), a silicone-based organic polymer, which is one of the most commonly used materials in bio-medical microdevices because of their easy fabrication process, biocompatibility, and low cost (Jose et al., 2007; Sia \& Whitesides, 2003). We first conduct the 
stereotaxic operation on mouse pups using the PDMS device and evaluate its utility. Finally, we discuss advantages of this PDMS device and future applications of our method.

\section{Materials and Methods}

\subsection{Animals}

All animal experiments were approved by the University of Tokyo and Toho University, and followed rules of the animal experiment committee of these universities. C57BL/6 mice were purchased from Sankyo Laboratory (Japan). Mice were maintained in clean cages with purified paper bedding (Alpha-Dri, Shepherd Specialty Papers, USA) under a 12-h light/dark cycle (lights-on 07:00) with food and water ad libitum. To reduce the body size differences, pups were culled to five at postnatal day (PND) 2 (Agnish \& Keller, 1997). Body weight was measured at PND 2 and 10. At PND 10, pups with culling showed increased body weight and decreased variation coefficient compared to those without culling (Fig. 1). All experiments were performed between 8:00 and 11:00.

\subsection{Fabrication of an anesthetic device with stereotaxic function}

In this study, we developed an anesthetic device with brain stereotaxic function using 3D scanned data from an actual head of a mouse pup. The overall fabrication process is shown in Fig. 2. First, a 10-day-old pup (6.1 g) was scanned to acquire the 3D CAD data using a 3D scanner (Hamano engineering, Japan) (Fig. 2a). This data was incorporated into the design of a single flow channel for anesthetic gas using 3D modeling software (Fig. 2b). The actual information for dimensions of our device was provided in Suppl. Fig. 1. After fabricating 3D molds using a 3D printer (KEYENCE, Japan), we transcribed the upper and lower PDMS (Torey, Japan) replicas from the molds and bonded them using etching system FA-1 (Samco, Japan) (Oxygen gas: 20 mL/min, Power: 50 W, Time: 5 s) (Fig. 2c). Two holes (4 mm in diameter) were made using biopsy punch (Fig. 2d). Finally, two tubes were inserted into these holes to connect to a commercial inhalation anesthesia apparatus (Muromachi, Japan). To measure how much force was necessary to remove a pup's head from the PDMS device, a tail of pup anesthetized by sodium pentobarbital was pulled toward horizontal direction using a force gauge (IMADA, Japan) (Suppl. Fig. 2a).

\subsection{Confirmation of anesthetic control by tail pinch}


PND 10 pups were gently handled and placed into the PDMS device by the experimenter and exposed to 1,2 , or $4 \%$ isoflurane. The tails were pinched using tweezers every $15 \mathrm{~s}$ for up to $10 \mathrm{~min}$. The time at which the pup stopped responding to the tail pinch was recorded.

\subsection{Tracer injection into mouse pup brain using the PDMS device}

PND 10 pups (6.1-6.2 g) were used for the brain microinjections. The PDMS device was incorporated within a commercially available stereotaxic apparatus (Narishige, Japan). First, the PDMS device (red arrows, Fig. 5a) was set at an orthogonal orientation to the ear bars (black arrowheads, Fig. 5a) in front. Next, the pup's nose and head were promptly placed into our device for head stabilization and anesthesia. After 2\% isoflurane anesthesia, the skull was exposed and a hole was drilled. For tracer injection, biotin-conjugated dextran amine (BDA) (Molecular Probes, USA) was dissolved in $0.1 \mathrm{M}$ phosphate buffered saline (PBS) ( $\mathrm{pH} 7.4$ ) to a final concentration of 5\%. Glass micropipettes with a tip diameter of 50-60 $\mu \mathrm{m}$ were prepared for the BDA injection. BDA was injected iontophoretically (5 $\mu \mathrm{A}, 7 \mathrm{~s}$ on/ $7 \mathrm{~s}$ off for $5 \mathrm{~min}$ ) at the stereotaxic coordinates $\mathrm{A}-2.5, \mathrm{~L} 3, \mathrm{~V} 4 \mathrm{on}$ the right hemisphere. After injection, skin was sutured and returned to the home cage with mother and siblings. After 3 days (PND 13), pups were sacrificed to confirm the injection.

\subsection{Preparation and staining of the brain sections}

Mice were deeply anesthetized by sodium pentobarbital injection (50 mg/kg, intraperitoneally) and transcardially perfused with $4 \%$ paraformaldehyde (PFA) in $0.1 \mathrm{M}$ PBS, pH 7.4. The brains were removed and additionally fixed in $4 \%$ PFA for $5 \mathrm{~h}$ on ice, and then placed in $30 \%$ sucrose in PBS overnight at $4{ }^{\circ} \mathrm{C}$. The brains were embedded in optimum cutting temperature (OCT) compound (Sakura FineTek, Japan), and 14- $\mu$ m coronal cryosections were prepared using a cryostat. For visualization of the BDA-labeled neurons, sections were rinsed with 0.1 M PBS, permeabilized with 0.3\% Triton X-100 in PBS, incubated with $0.3 \% \mathrm{H}_{2} \mathrm{O}_{2}$ in methanol for $1 \mathrm{~min}$, and then in $\mathrm{ABC}$ peroxidase reagent (Vectastain ABC Elite kit, Vector Laboratories, USA) for $1 \mathrm{~h}$ according to the manufacturer's protocol. The labeling was visualized under a microscope after incubating the sections in 3, 3-diaminobenzidine solution (Nakarai, Japan) for $15 \mathrm{~min}$.

\subsection{Statistical analyses}


Student's t-test was performed with significance set at $p<0.05$. All statistical analyses were conducted using R2.9.0 (R Development Core Team (http://www.R-project.org)).

\section{Results}

The PDMS device was fabricated using data from a PND 10 pup as described in Materials and Methods section 2.2 (Fig. 2). We evaluated the PDMS devise for stabilization, anesthetic control, and stereotaxic operation. The head of a pup was placed in the PDMS device and its nose was fitted into the nose poke hole (Fig. 3a). Even upon tilting the devise, the head fitted well and the pup did not fall off the PDMS device (Fig. 3b). When the PDMS device was set vertically, the pup fell off the device meaning that the holding force of the device was less than the pup's own body weight. The head of a pup remained in the PDMS device after pulling the pup with $0.056 \mathrm{~N}(\mathrm{n}=3, \mathrm{SD}=0.001)$ that corresponded to $93.22 \%$ of own body weight, and started removing from the device at $0.058 \mathrm{~N}(\mathrm{n}=3$, $\mathrm{SD}=0.001,96 \%$ of own body weight) (Suppl. Fig. 2b). To confirm the anesthetic control, we checked the responses of the pup to the tail-pinch test, across different isoflurane concentrations (Fig. 4). Pups exposed to $1 \%$ isoflurane showed continuous nociceptive responses. With $2 \%$ isoflurane, which is normally used for inhalation anesthesia, pups showed no response to the tail-pinch after 135-180 s exposure. Pups exposed to $4 \%$ isoflurane were anesthetized quickly within $90 \mathrm{~s}$. None of the pups died during or after this examination. The PDMS device was autoclaved or washed with $70 \%$ ethanol after use. Finally, we performed stereotaxic operation using our device (Fig. 5). The PDMS device was applied in combination with a commercially available stereotaxic apparatus (Fig. 5a). The PDMS device was also successfully connected to a commercially available anesthetic apparatus via two tubes for inlet and outlet ports (Fig. 5a). After the start of inhalation anesthesia using $2 \%$ isoflurane, the head of the pup was put into the PDMS device. Since the head was fitted to the stereotaxic part, we were able to make a small incision and a drilled hole in the skull without vibration. A BDA-filled glass capillary was inserted into the hole (Fig. 5b). After 3 days, the histological staining revealed that BDA injection was possible using our PDMS device (Fig. 5c).

\section{Discussion}

In the previous studies, while a miniaturized stereotaxic instrument for surgery in rat pups was developed, it required a wide incision in the head skin to expose the membranous external acoustic meatus for stabilization (Cunningham \& McKay, 1993). Plasticine clay had been used to fabricate a 
mold for non-invasive stabilization (Hoorneman, 1985). The clay mold had poor sanitary control and less flexibility in combination with other experimental equipment such as an inhalation anesthetic apparatus. The clay mold was also unsuitable for mass production. In contrast, the PDMS device presented here overcomes those problems and has additional advantages. First, our device is capable of stabilizing the pup head in a non-invasive manner. Since the skull of a pup is very soft, the PDMS device needs to avoid holding the head too tight. Although the PDMS device is unable to support pup's own body weight, our data indicate the holding force of the device corresponded to $93 \%$ of own weight is sufficient to stabilize the head for brain surgery. Second, because PDMS is autoclavable and washable, it is easy to maintain sterilized conditions. Third, PDMS is soft enough to cut and make a hole. Our device is compatible with various types of commercial stereotaxic and anesthetic apparatuses via trimming and tube insertion, respectively. Fourth, the PDMS device is suitable for mass production since it is replicated from 3D CAD data. Finally, additional advantages include reduction in the number of animals used. Because 3D scanned data can be taken from lightly anesthetized pups, there are no needs to sacrifice only for making the mold. Moreover, the PDMS device is simple to use and contributes to reducing the operation time. The present study indicates that our device can stabilize the pup's head with the non-invasive manner and functions as an anesthetic device that can be sterilized.

By applying the present fabrication method, it is possible to easily prepare an anesthetic device equipped with stereotaxic function for various brain sizes. Though the PDMS device has need to be newly fabricated according to pup's size, our method is capable of rapid fabrication of the device within 2 days at a low price from the scanning to the PDMS bonding. There are also outsourcing services for the scanning or $3 \mathrm{D}$ printing. A device fabrication for specific operations as presented here will be a

major strategy in neuroscience research of animal behavior. Appropriate instruments for stereotaxic operation on mouse pups will facilitate in the studies on understanding of molecular basis of behavioral development in combination with genetic engineering such as targeted injection of vectors and drugs into mutant pups.

\section{Acknowledgement}

The authors acknowledge the technical support provided by Saori Mori and Maiko Onuki (The University of Tokyo). This work was supported by Health and Labour Sciences Research Grants, JSPS KAKENHI Grant Number 25861038 and JST PRESTO program. 


\section{References}

Agnich ND \& Keller KA. The rational for culling of rodent litters. Toxicological Sciences. 2007, 38, 2-6.

Cetin A, Komai S, Eliava M, Seeburg P, Osten P. Stereotaxic gene delivary in the rodent brain. Nature Protocols. 2007,1, 3166-3173.

Cunningham MG, McKay RD. A hypothermic miniaturized stereotaxic instrument for surgery in newborn rats. Journal of neuroscience methods. 1993; 47: 105-14.

Flecknell PA. Laboratory animal anaesthesia. An introduction for research workers and technicians: Academic Press, 1987.

Hoorneman E. Stereotaxic operation in the neonatal rat; a novel and simple procedure. Journal of neuroscience methods. 1985, 14, 109-16.

Jose NM, de Almeida Prado LA, Schiavon MA, Redondo SU, Yoshida IV. Partially pyrolyzed poly (dimethylsiloxane) - based networks: Thermal characterization and evaluation of the gas permeability. Journal of Polymer Science Part B: Polymer Physics. 2007, 45, 299-309.

Tsuneoka Y, Maruyama T, Yoshida S, Nishimori K, Kato T, Michael Numan, KumiO. Kuroda. Functional, anatomical, and neurochemical differentiation of medial preoptic area subregions in relation to maternal behavior in the mouse. Journal of Comparative Neurology. 2013, 52, 1633-1663.

Sia SK, Whitesides GM. Microfluidic devices fabricated in poly (dimethylsiloxane) for biological studies. Electrophoresis. 2003; 24: 3563-76.

\section{Fig. 1}

Body weight comparison of postnatal day 10 pups between without (-) and with (+) culling. All numerical data are shown as mean \pm SD. $* * p<0.01 . n=24(-), 15(+)$. Coefficient of variation: 10.66 $(-), 8.95(+)$.

\section{Fig. 2}

Fabrication flow of an inhalation anesthetic device for mouse pups equipped with a brain stereotaxic instrument. (a) A day 10 pup (red arrow) was scanned to obtain 3D CAD data. (b) The shape of the scanned head part (red arrow) was subtracted from the design of the device (black arrowhead). (c) After fabricating molds using a 3D printer, upper and lower polydimethylsiloxane (PDMS) replicas were transcribed and bonded. The dotted circle shows the structure based on a real pup head. (d) Two tubes 
are inserted to acquire inlet and outlet ports. The device consists of a single flow channel for anesthetic gas having a nose poke hole and a brain stereotaxic part.

\section{Fig. 3}

Confirming the fitting of the pup head into the PDMS device. (a) The head of a PND 10 pup was put into the device and its nose fitted into the nose poke hole. (b) The PDMS device was tilted to examine whether the pup would fall off or not.

\section{Fig. 4}

Confirmation of the anesthetic control. PND 10 pups were exposed to 1,2, and $4 \%$ isoflurane with tail pinches every $15 \mathrm{sec}$. The times at which the pups stopped responding to the tail pinch were recorded and compared. $* * p<0.01 . \mathrm{n}=5$ in each condition. NA, not applicable.

\section{Fig. 5}

Microinjection into a pup brain using the PDMS device. (a) The PDMS device (red arrows) was incorporated in a commercially available typical stereotaxic apparatus. The device was set in orthogonal orientation to ear bars in front (black arrowheads). Two ports of the device were connected to an inhalation anesthesia apparatus. Biotinylated dextran amine (BDA) was filled into the glass capillary. (b) The head of a PND 10 pup was placed into the device and its nose fitted into a nose poke hole. After making a small hole on the skull, the glass capillary was inserted into the hole for BDA injection. (c) An example of histological staining showing BDA injection using the PDMS device. The right panel showed a high-magnification image of a square in the left panel. BLP, basolateral amygdaloid nucleus posterior part; LEnt, lateral entorhinal cortex; PMCo, posteromedial cortical amygdaloid nucleus; Py, pyramidal tract. 
Figure 1

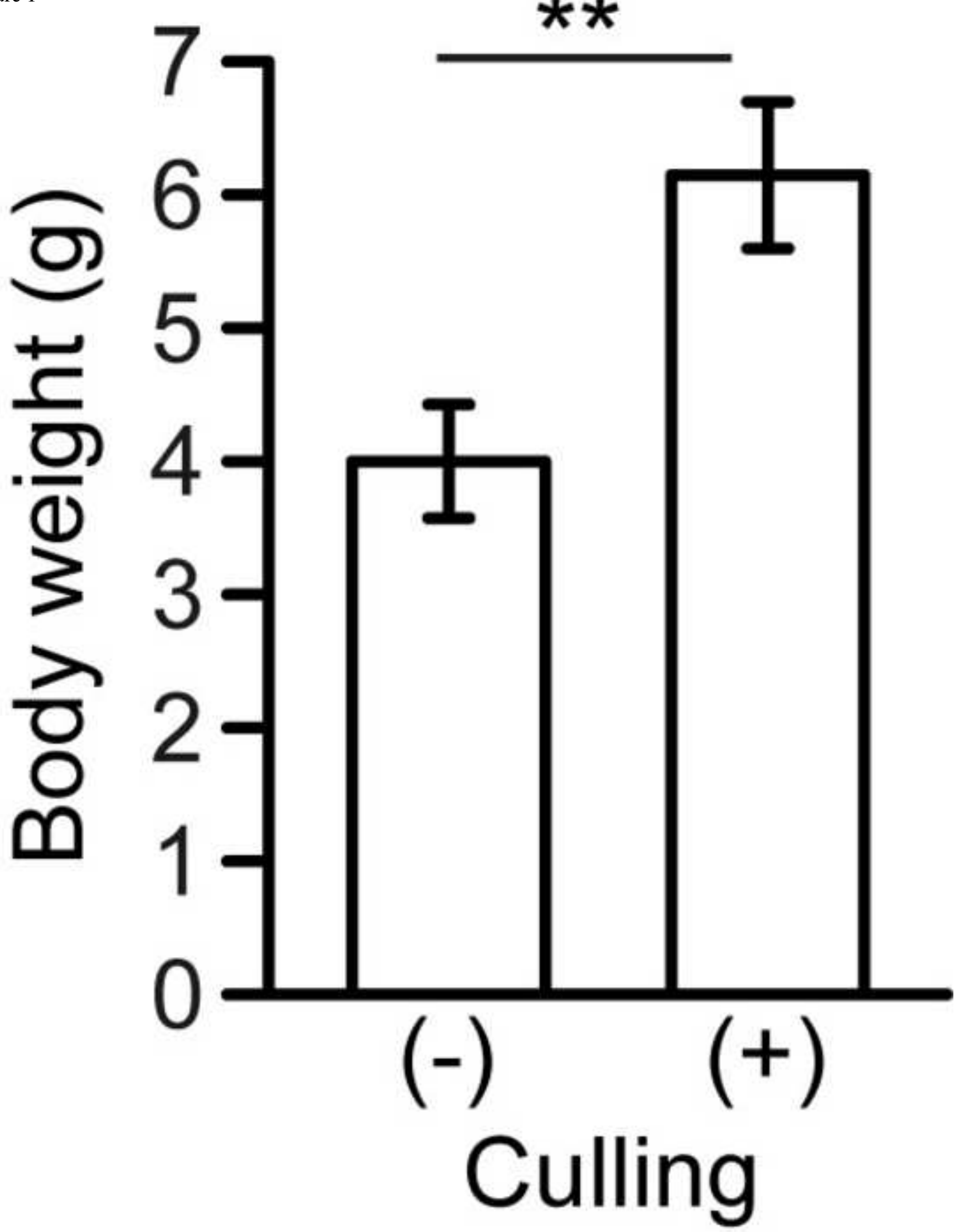


(a)

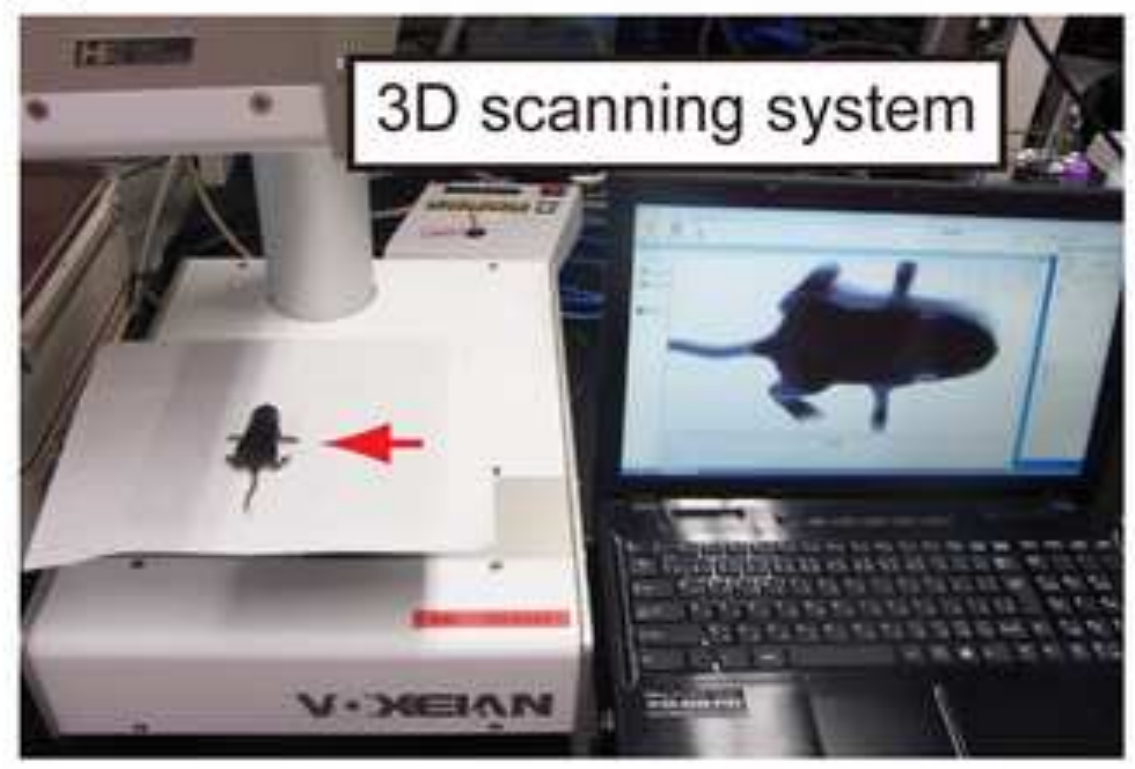

(c)

Upper replica
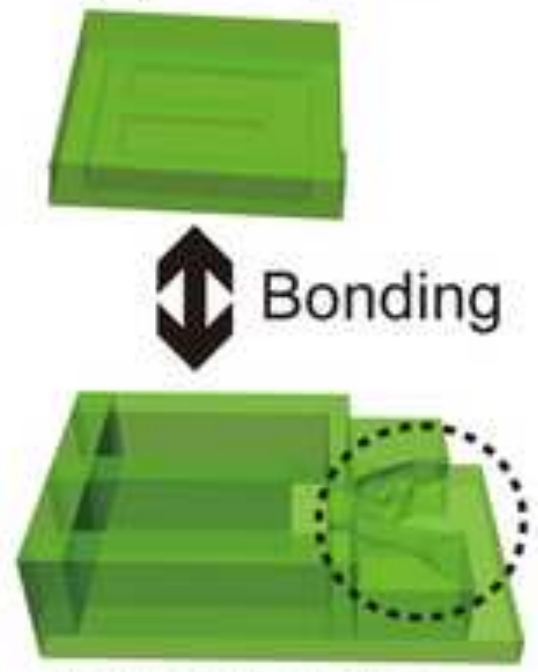

Lower replica (b)

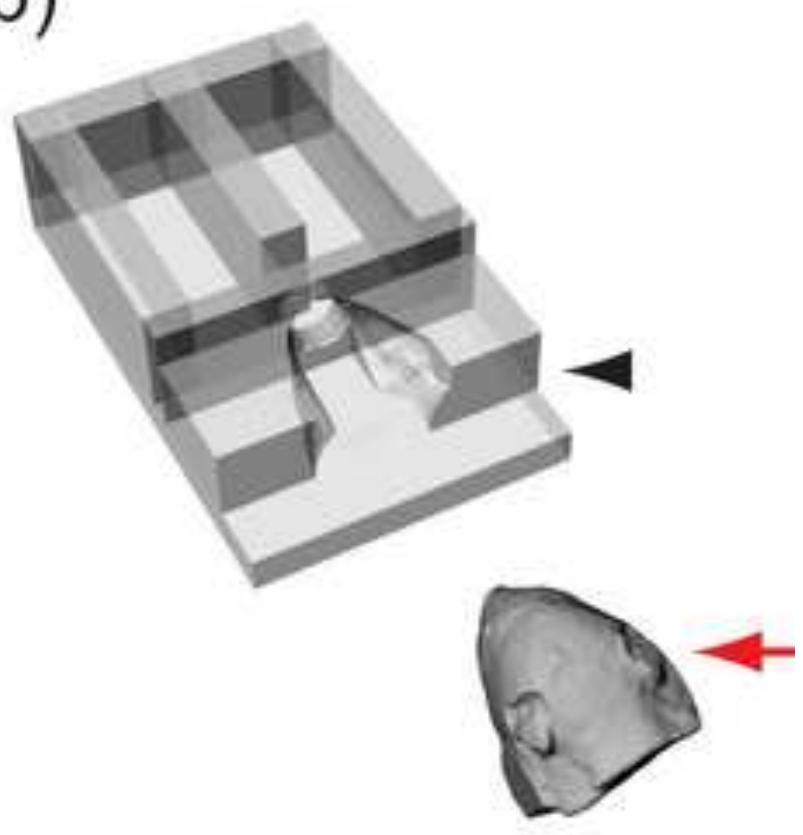

(d)

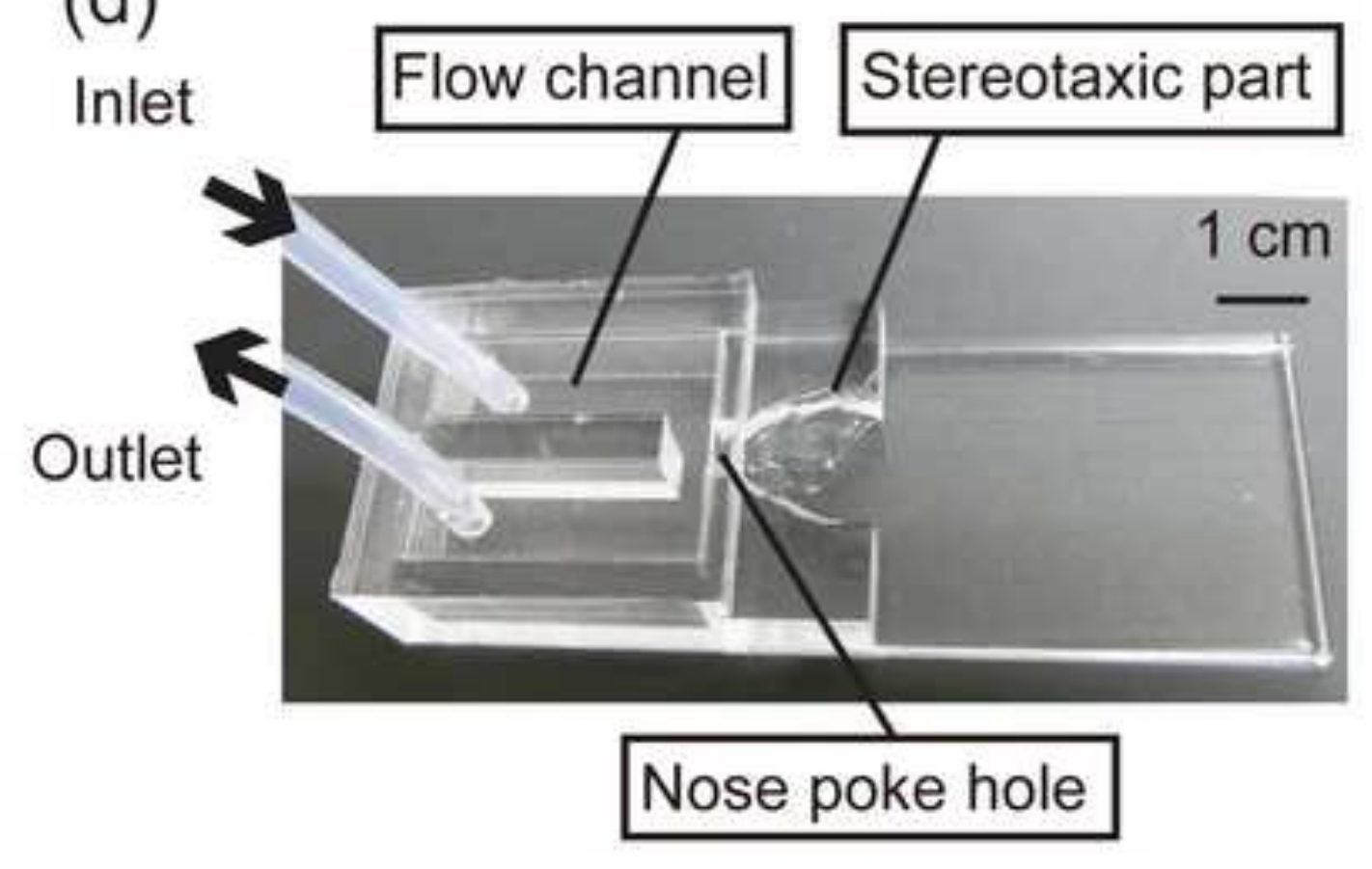



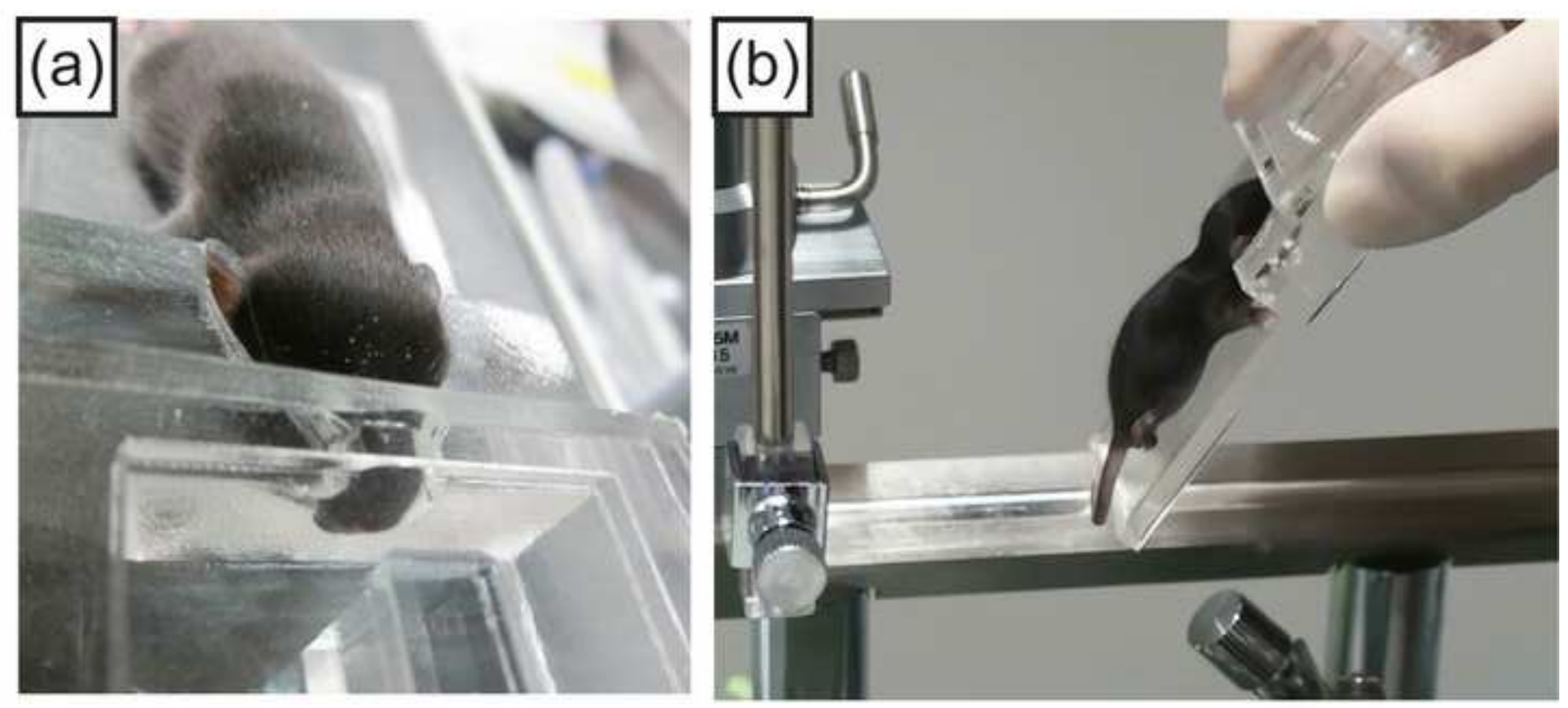
Figure 4

$$
2407
$$

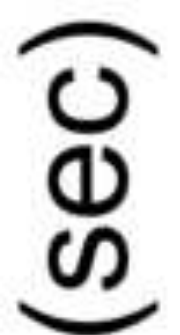

E

180

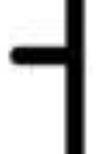

을 $120-$

듬

(1)

$\varepsilon$

$60-$

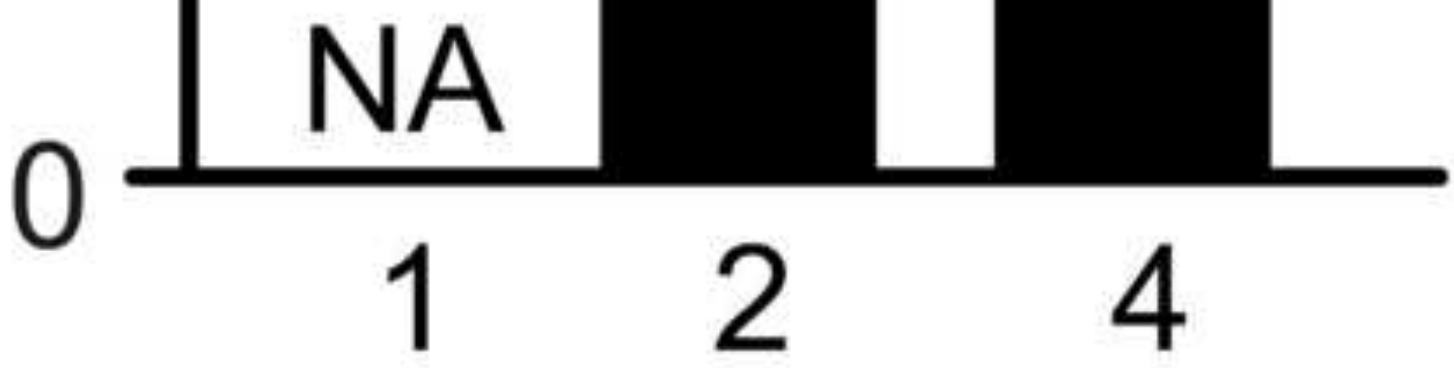

Concentration (\%) 
Figure 5

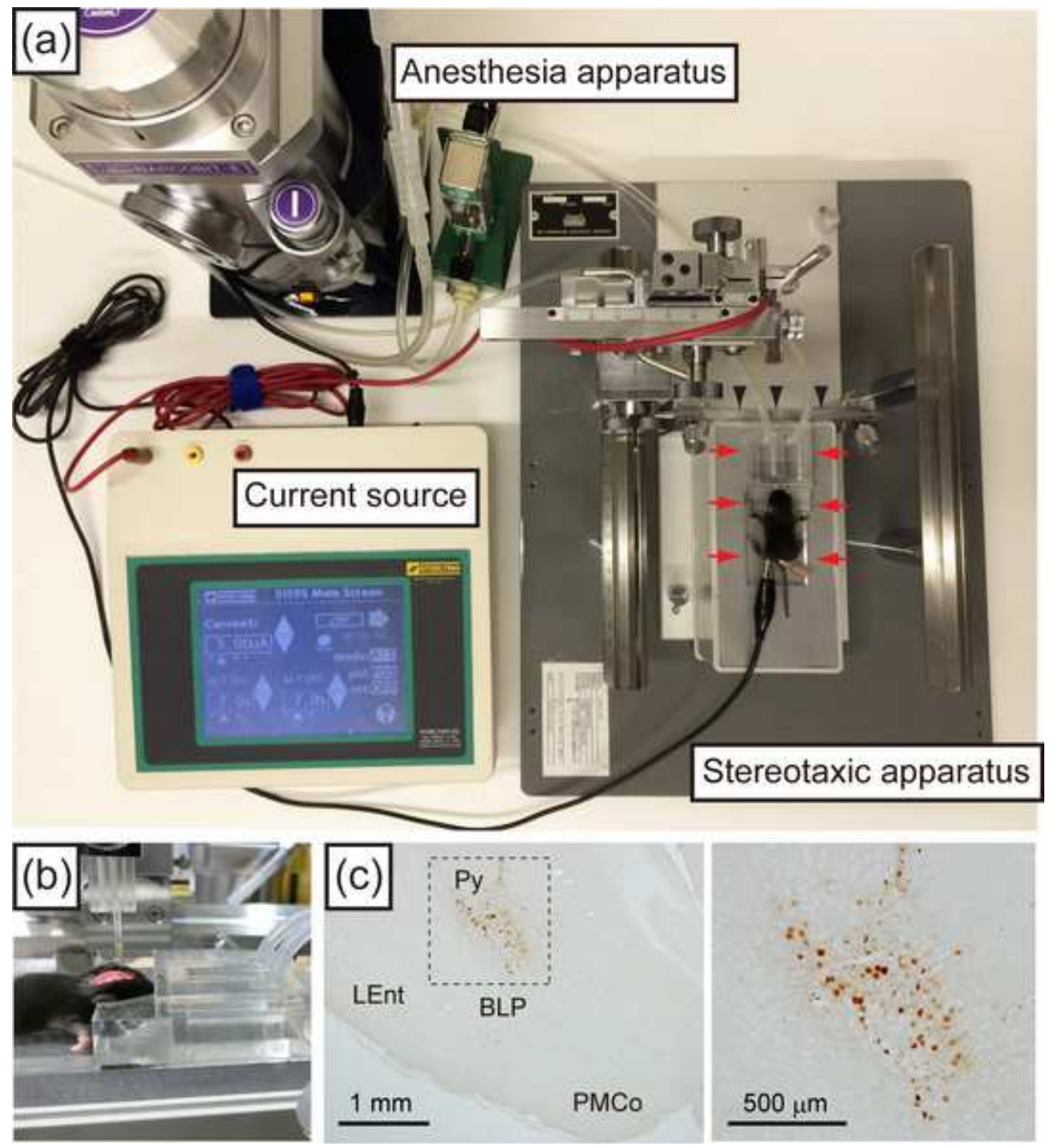

